\title{
Perturbation Theory-Aided Learned Digital Back-Propagation Scheme for Optical Fiber Nonlinearity Compensation
}

\author{
Xiang Lin, Shenghang Luo, Sunish Kumar Orappanpara Soman, Octavia A. \\ Dobre, Lutz Lampe, Deyuan Chang, Chuandong Li
}

\begin{abstract}
Derived from the regular perturbation treatment of the nonlinear Schrödinger equation, a machine learning-based scheme to mitigate the intra-channel optical fiber nonlinearity is proposed. Referred to as the perturbation theory-aided (PA) learned digital back propagation (LDBP), the proposed scheme constructs a deep neural network (DNN) in a way similar to the split-step Fourier method: linear and nonlinear operations alternate. Inspired by the perturbation analysis, the intra-channel cross phase modulation term is conveniently represented by matrix operations in the DNN. The introduction of this term in each nonlinear operation considerably improves the performance, as well as enables the flexibility of PA-LDBP by adjusting the numbers of spans per step. The proposed scheme is evaluated by numerical simulations of a single-carrier optical fiber communication system operating at $32 \mathrm{Gbaud}$ with 64-quadrature amplitude modulation and $20 \times 80 \mathrm{~km}$ transmission distance. The results show that the proposed scheme achieves approximately $3.5 \mathrm{~dB}, 1.8 \mathrm{~dB}, 1.4 \mathrm{~dB}$, and $0.5 \mathrm{~dB}$ performance gain in terms of $Q^{2}$ factor over the linear compensation, when the numbers of spans per step are 1, 2, 4, and 10, respectively. Two methods are proposed to reduce the complexity of PA-LDBP, i.e., pruning the number of perturbation coefficients and chromatic dispersion compensation in the frequency domain for multi-span per step cases. Investigation of the performance and complexity suggests that PA-LDBP
\end{abstract}

Xiang Lin and Octavia A. Dobre are with Faculty of Engineering and Applied Science, Memorial University, St. John's, NL, A1B 3X5, Canada; Shenghang Luo and Lutz Lampe are with Department of Electrical and Computer Engineering, University of British Columbia, Vancouver, BC V6T 1Z4, Canada; O. S. Sunish Kumar was with the Department of Electrical and Computer Engineering, the University of British Columbia, Vancouver, BC V6T 1Z4, Canada, and he is now with the School of Engineering, Ulster University, Jordanstown, BT37 OQB, UK; Deyuan Chang and Chuandong Li are with the Huawei Technologies Canada, Ottawa, ON K2K 3J1, Canada. Corresponding email: xiang.lin@mun.ca 
attains improved performance gains with reduced complexity when compared to LDBP in the cases of 4 and 10 spans per step.

\section{INTRODUCTION}

Our digitally-interconnected world relies heavily on optical fiber communications to transport the majority of tremendous information. Over the past decades, digital signal processing (DSP) has played an essential role to achieve reliable and high-speed optical fiber communications [1]-[6]. To date, most of the linear impairments, such as chromatic dispersion (CD) and polarization mode dispersion, have been studied extensively and addressed well by DSP algorithms [7], [8]. Fiber nonlinearity, on the other hand, appears to be the dominant barrier that limits the performance of today's optical fiber communication systems. The power-dependent nature of the fiber nonlinear effect restricts the maximum launch power into the optical fiber, and hence, limits the effective signal-to-noise ratio (SNR) at the receiver. To mitigate the nonlinear effects, several DSP techniques have been proposed, such as the digital back-propagation (DBP) and perturbation theory-based (PB) nonlinearity compensation (NLC) [9]-[13]. DBP attempts to compensate the deterministic fiber nonlinearity at the receiver by emulating the signal propagation in the fiber channel in a reverse direction. Its complexity is significantly higher than linear compensation. PB-NLC is typically developed based on the first-order perturbation of the nonlinear Schrödinger equation (NLSE). The PB-NLC technique exhibits reduced computational complexity in comparison with DBP. A number of variants based on DBP and PB-NLC have been developed to strike a balance between performance and complexity [14]-[16]. Nonetheless, the sophisticated nature of the distortion caused by the interaction between the $\mathrm{CD}$, fiber nonlinearity, and amplified spontaneous emission noise have made it challenging to develop a NLC DSP algorithm to achieve good performance and low complexity suitable for commercial implementation.

Recently, the potential of machine learning to overcome fiber nonliearity has been explored in the literature [17]-[21]. In general, these techniques seek to establish a mapping between the input and output through training, and the learned mapping is employed at the inference stage to remove nonlinear effects. For example, in learned PB-NLC, the data triplets are used 
as input, and the output is the additive nonlinear distortion field [21]. The mapping is obtained through training, using a large amount of data instead of rigorous mathematical reasoning; as such, it typically requires empirical experience to find out the model hyper-parameters [22]. Unlike such "black-box" solutions, a technique that employs a deep neural network (DNN) to unfold the conventional DBP, referred to as the learned DBP (LDBP), has been proposed recently [23], [24]. LDBP interprets the split-step Fourier method (SSFM) as a DNN with each hidden layer as the linear section and the corresponding activation function as the nonlinear section. As a result, LDBP incorporates the optimization capability of a DNN into the conventional DBP by parametrizing the SSFM and training the parameters through supervised learning. Performance improvement and complexity reduction compared to the conventional DBP are achieved. Additionally, this technique leads to explicit hyper-parameters selection, such as the number of layers and the activation function. This approach has been also validated through experiments [25], [26]. More recently, a convolutional neural network (CNN) has been considered to replace the DNN structure in LDBP [27]. To summarize, the LDBP technique accomplishes the linear steps through the weight matrices operation in DNN or CNN, and the nonlinear steps through the nonlinear activation functions. However, the number of layers in the DNN increases with the number of fiber spans in the link, and such a deep structure faces several challenges, such as the extended training time/memory and the well-known vanishing gradient problem [22].

In this paper, we propose a novel design, where the nonlinear steps of LDBP are improved with the aid of the first-order perturbation analysis. One may see that the proposed perturbationaided (PA)-LDBP scheme shares some merits with the enhanced SSFM (ESSFM), where the nonlinearity compensation requires the adjacent symbols of the current symbol of interest. However, the differences between the proposed PA-LDBP and ESSFM in [24] and [27] are evident. Specifically, in [24], the focus is on the performance gain of LDBP that adopts ESSFM over the conventional DBP with ESSFM; while in [27], the aim is to improve the performance at each nonlinear step, and only 1 span per step is considered. In this work, we show that the benefits of our proposed PA-LDBP are fourfold:

- The perturbation analysis is employed to facilitate the DNN initialization. This physics- 
informed initialization guides the training deterministically towards a promising solution, and thus, significantly reduces the training effort.

- It provides a flexible structure which enables a multi-span per step configuration.

- It outperforms LDBP for the same number of spans per step.

- It achieves a reduction in complexity when multi-span per step is considered.

The remainder of this paper is organized as follows. In Section II, the system model, the LDBP, and the proposed PA-LDBP scheme are introduced. In Section III, a comprehensive performance analysis is carried out, the performance of PA-LDBP is compared to that of LDBP, and the initialization for PA-LDBP is discussed. In Section IV, the complexity analysis is provided, and two approaches to reduce the overall complexity are presented. Finally, conclusions are drawn in Section V.

Notations: Throughout the paper, the upper case bold letters represent matrices, while the lower case bold font denotes column vectors. $\mathbf{A}^{T}$ indicates the transpose of matrix $\mathbf{A}$, and $\mathbf{A}^{*}$ denotes the conjugate of matrix $\mathbf{A}$. $\mathbb{C}$ represents the complex-valued domain, and $\mathbb{Z}$ represents the integer domain.

\section{The PROPOSED PA-LDBP SCHEME}

In this section, the system model is introduced, followed by a brief description of the LDBP technique. Then, details of the proposed PA-LDBP are provided.

\section{A. System Model}

In a single mode optical fiber, the propagation of the optical field envelope $u$ at the retarded time frame $t$ and distance $z$ is governed by the scalar NLSE, which is given as

$$
\frac{\partial}{\partial z} u(z, t)+\frac{\alpha}{2} u(z, t)+j \frac{\beta_{2}}{2} \frac{\partial^{2}}{\partial t} u(z, t)=j \gamma|u(z, t)|^{2} u(z, t),
$$

where $\alpha$ is the attenuation coefficient, $\beta_{2}$ is the group velocity dispersion coefficient, and $\gamma$ is the nonlinearity coefficient [28], [29]. The signal launched into the optical fiber channel is given by

$$
u(0, t)=\sqrt{P} \sum_{n=-\infty}^{\infty} s_{n} g(0, t-n T)
$$


where $P$ is the launch power, $s_{n}$ is the $n$th symbol, $g(0, t-n T)$ is the pulse, and $T$ is the symbol duration. The symbol sequence is assumed independent and identically distributed and with unit power. After propagating through the optical channel, the signal is coherently detected. Then, it passes through a low-pass filter and is sampled at $t=n T, n \in \mathbb{Z}$. Subsequently, a sampled sequence, $\mathbf{x}=\left[x_{1}, x_{2}, \ldots, x_{N}\right]^{T}$, is obtained. The objective of NLC techniques is to recover information from $\mathbf{x}$.

\section{B. The LDBP Technique}

DBP can be viewed as a concatenation of linear and nonlinear steps. In the absence of noise, the transmitted signal is estimated by reversing the NLSE:

$$
\frac{\partial}{\partial z} u(z, t)=\left(\hat{D}^{-1}+\hat{N}^{-1}\right) u(z, t)
$$

where $\hat{D}=-j \frac{\beta_{2}}{2} \frac{\partial^{2}}{\partial t^{2}}-\frac{\alpha}{2}$ and $\hat{N}=j \gamma|u(z, t)|^{2}$ are the linear and nonlinear operators, respectively, and $(\cdot)^{-1}$ is the reverse operation [29]. Given the sampled signal, $\mathbf{x}$, the linear step of DBP is expressed as

$$
\mathbf{x}^{\mathrm{CD}}=\exp \left(\frac{\alpha}{2} \mu\right) \mathbf{F}^{-1}\left(\mathbf{F} \mathbf{x} \exp \left(-j \frac{\beta_{2}}{2} \boldsymbol{\omega}^{2} \mu\right)\right)
$$

where $\mathbf{F}$ is the $N \times N$ discrete Fourier transform (DFT) matrix, $\mathbf{F}^{-1}$ is the inverse DFT matrix, $\mu$ is the step size, and $\boldsymbol{\omega}$ is the DFT angular frequency, whose $i$ th element is given by $\omega_{i}=2 \pi f_{i}$ $\left(f_{i}=f_{s}(i-1) / N\right.$ if $i<N / 2$ and $f_{i}=f_{s}(i-1-N) / N$ if $\left.i \geq N / 2\right) . f_{s}$ is the sampling rate. After that, a nonlinear operation is performed sample-by-sample in time domain according to

$$
x_{n}^{\mathrm{NL}}=x_{n}^{\mathrm{CD}} \exp \left(-j \zeta \gamma L_{\mathrm{eff}}(\mu)\left|x_{n}^{\mathrm{CD}}\right|^{2}\right)
$$

where $x_{n}^{\mathrm{NL}}$ is the $n$th sample after the nonlinear step, $\zeta$ is a parameter which needs to be optimized empirically, $L_{\mathrm{eff}}(\mu)=(1-\exp (-\alpha \mu) / \alpha)$ is the effective nonlinear length, and $x_{n}^{\mathrm{CD}}$ is the $n$th sample in $x^{C D}$. Typically, a smaller step size leads to an enhanced performance but results in higher complexity.

LDBP employs the connection between the DBP and a DNN: in both cases, linear and nonlinear steps alternate. In LDBP, all linear steps are parametrized as the DNN weight matrices 
$\mathbf{W}^{(\mathbf{1})}, \ldots \mathbf{W}^{(\ell)}, \ldots \mathbf{W}^{(L)}$, where $\mathbf{W}^{(\ell)} \in \mathbb{C}$ is an $N$-by- $N$ matrix, the superscript $(\ell)$ represents the $\ell$ th step, and $L$ represents the number of steps. On the other hand, the nonlinear operation at the $\ell$ th step is performed by applying the activation function

$$
\sigma^{(l)}\left(x_{n}^{\mathrm{CD}}\right)=x_{n}^{\mathrm{CD}} \exp \left(-j \eta^{(\ell)} \gamma L_{\mathrm{eff}}(\mu)\left|x_{n}^{\mathrm{CD}}\right|^{2}\right)
$$

in the DNN, where $\eta^{(\ell)}$ corresponds to $\zeta$ in (5) and is configured as a non-trainable parameter in LDBP [24]. By concatenating all the steps, LDBP can be expressed concisely as

$$
\Pi_{\mathrm{LDBP}}(\mathbf{x})=\sigma\left(\mathbf{W}^{(L)} \ldots \sigma\left(\mathbf{W}^{(1)} \mathbf{x}\right)\right)
$$

A parameter set $\left(\mathbf{W}^{(1)}, \ldots, \mathbf{W}^{(\ell)}, \ldots, \mathbf{W}^{(L)}\right)$ is defined, which is optimized through supervised learning. It is worth noting that the rows of the linear matrix $\mathbf{W}^{(\ell)}$ are circularly-shifted versions of $\mathbf{h}^{(\ell)}=\left(h_{-V}^{(\ell)}, \ldots, h_{-1}^{(\ell)}, h_{0}^{(\ell)}, h_{1}^{(\ell)}, \ldots, h_{V}^{(\ell)}, 0, \ldots, 0\right)$, where $h_{v}^{(\ell)} \in \mathbb{C}, v=-V, \ldots, V$ represent the finite impulse response (FIR) filter coefficients for CD compensation [24]. Note that the filter is symmetric, so the trainable parameters at the $\ell$ th step are $\mathbf{h}_{\mathbf{v}}{ }^{(\ell)}=\left(h_{0}^{(\ell)}, h_{1}^{(\ell)}, \ldots, h_{V}^{(\ell)}\right)$. Thus, the parameter set for the entire model is downsized to $\boldsymbol{\theta}=\left\{\mathbf{h}_{\mathbf{v}}{ }^{(1)}, \ldots, \mathbf{h}_{\mathbf{v}}{ }^{(\ell)}, \ldots, \mathbf{h}_{\mathbf{v}}{ }^{(L)}\right\}$. Recently, a CNN structure has been employed to replace the DNN structure [27]. The CNNbased LDBP considers a block of symbols as the input, while the output is the equalized symbol corresponding to the center of the input block. In spite of different structures, both DNN and CNN perform circular convolutions-based time-domain equalization (TDE) to compensate for the $\mathrm{CD}$ at each step.

LDBP offers important advantages over other machine learning-based NLC methods which are data-driven [18], [21]. Firstly, the DNN structure is based on physical principles, which is the SSFM approximation of the signal propagation over optical fibers. It follows that hyperparameters, such as the type of the activation function and the number of layers, are associated with physical parameters and their choice or optimization are interpretable similar to the case of conventional DBP. Secondly, the initialization of the DNN weights for linear steps can be based on analytical results used in the DBP, which greatly facilitates successful and fast training. 


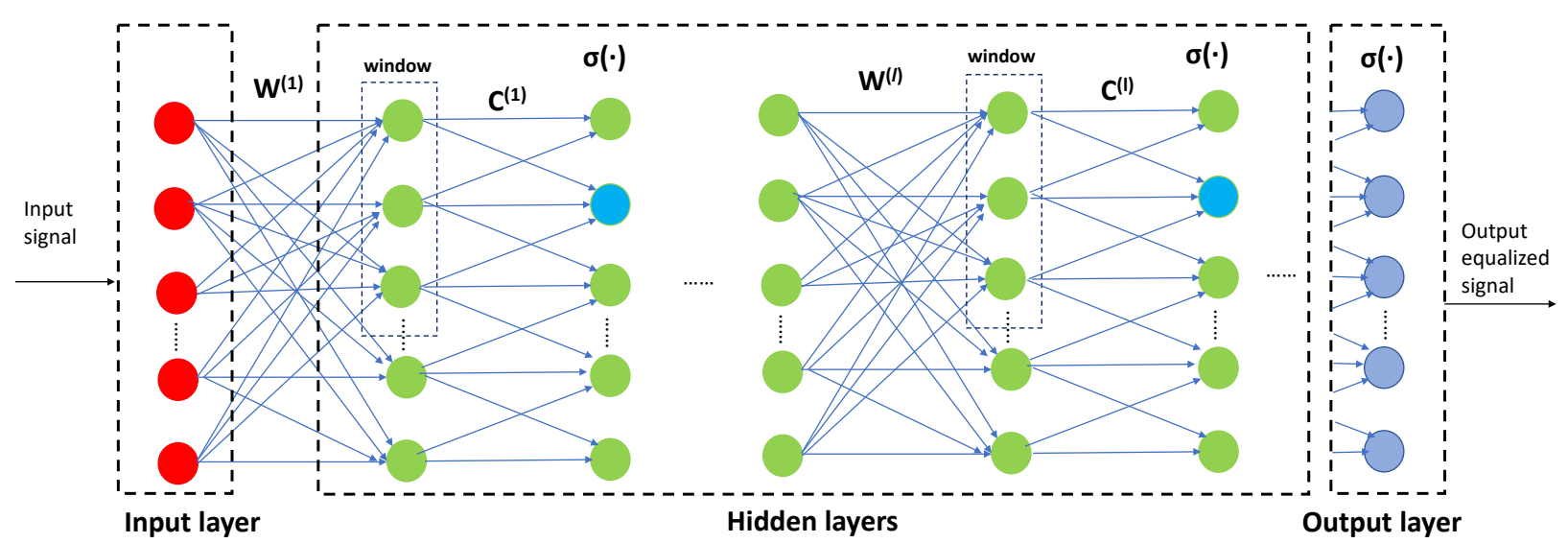

Fig. 1: PA-LDBP structure.

These benefits will be inherited by the proposed PA-LDBP scheme.

\section{The PA-LDBP Scheme}

LDBP has been shown to substantially improve the performance and complexity when compared to the conventional DBP [24]. An important factor in the complexity reduction of LDBP is the pruning of network parameters, i.e., the number of non-zero elements of the weight matrices $\mathbf{W}^{(l)}$, which effectively reduces the number of coefficients of the TDE filter [30]. However, it is noticed that most of the investigations of the LDBP scheme consider self phase modulation (SPM), and thus, a relatively small number of spans per step is required. Alternative speaking, the number of layers in LDBP needs to increase with the number of fiber spans for successful nonlinearity compensation. Hence, A very deep DNN is required, which is challenging [22].

To overcome the above-mentioned problems, we propose to refine the nonlinearity compensation by considering both SPM and intra-channel cross-phase modulation (IXPM). The details are presented in the following.

Without loss of generality, we consider the first step of PA-LDBP, and the first-order nonlinear distortion to the $n$th sample is given as

$$
\Delta_{n}=j P^{\frac{3}{2}} \sum_{m, k} x_{k}^{\mathrm{CD}}\left(x_{m+k}^{\mathrm{CD}}\right)^{*} x_{m}^{\mathrm{CD}} C_{m, k},
$$


where $C_{m, k}$ is the perturbation coefficient that can be obtained by (9) shown at the bottom of the page, with $f(z)$ as the power profile function defined as $\exp (-\alpha z)$ [10], [31]. It is worth noting that the distortion for other steps can be calculated in a similar way using (8). By expanding (8) and performing some algebraic simplifications, the distortion field can be separated into the SPM, IXPM, and intra-channel four-wave mixing (IFWM) effects, as follows:

$$
\Delta_{n}=j P^{\frac{3}{2}} x_{n}^{\mathrm{CD}}\left[\left|x_{n}^{\mathrm{CD}}\right|^{2} C_{0,0}+2 \sum_{k \neq 0}\left|x_{k}^{\mathrm{CD}}\right|^{2} C_{0, k}\right]+j P^{\frac{3}{2}} \sum_{m \neq 0, k \neq 0} x_{k}^{\mathrm{CD}}\left(x_{m+k}^{\mathrm{CD}}\right)^{*} x_{m}^{\mathrm{CD}} C_{m, k} .
$$

Let $\phi_{n}=P^{\frac{3}{2}}\left[\left|x_{n}^{\mathrm{CD}}\right|^{2} C_{0,0}+2 \sum_{k \neq 0}\left|x_{k}^{\mathrm{CD}}\right|^{2} C_{0, k}\right]$; then, when SPM and IXPM are both considered, the nonlinear step in PA-LDBP can be represented as

$$
\begin{aligned}
\sigma\left(x_{n}^{\mathrm{CD}}\right) & =x_{n}^{\mathrm{CD}}-\Delta_{n} \\
& \approx x_{n}^{\mathrm{CD}}\left(1-j \phi_{n}\right) \\
& \approx x_{n}^{\mathrm{CD}} \exp \left(-j \phi_{n}\right) \\
& =x_{n}^{\mathrm{CD}} \exp \left(-j P^{\frac{3}{2}}\left(\mathbf{x}_{k}^{\mathrm{CD}}\right)^{T} \mathbf{c}_{0}\right)
\end{aligned}
$$

where $\mathbf{x}_{k}^{\mathrm{CD}}$ is the vector $\left[\left|x_{n-k}^{\mathrm{CD}}\right|^{2}, \ldots,\left|x_{n}^{\mathrm{CD}}\right|^{2}, \ldots,\left|x_{n+k}^{\mathrm{CD}}\right|^{2}\right]^{T}$ and $\mathbf{c}_{\mathbf{0}}$ represents the perturbation coefficients vector which accounts for the SPM and IXPM effects, i.e., $\left[2 C_{0, k}, \ldots, C_{0,0}, \ldots, 2 C_{0, k}\right]^{T}$. Note that (11) holds when $\phi_{n} \ll 1$. By carefully examining (11), it can be seen that PA-LDBP involves one additional operation when compared to LDBP at each nonlinear step. This operation aims to learn the nonlinearity interaction between a number of adjacent samples. To generalize, we consider the $\ell$ th step, which includes 2 sets of weights: $\mathbf{W}^{(\ell)}$ and $\mathbf{C}^{(\ell)}$. The former can be configured in the same way as in LDBP for CD compensation; the rows of the latter are circularly-shifted versions of $\mathbf{c}_{\mathbf{0}}{ }^{(\ell)}$. By concatenating all the steps, one can summarize the PA-

$$
C_{m, k}=\frac{1}{T} \int_{0}^{z} d z \gamma f(z) \int d t g^{*}(z, t) g(z, t-m T) g(z, t-k T) g^{*}(z, t-(m+k) T)
$$




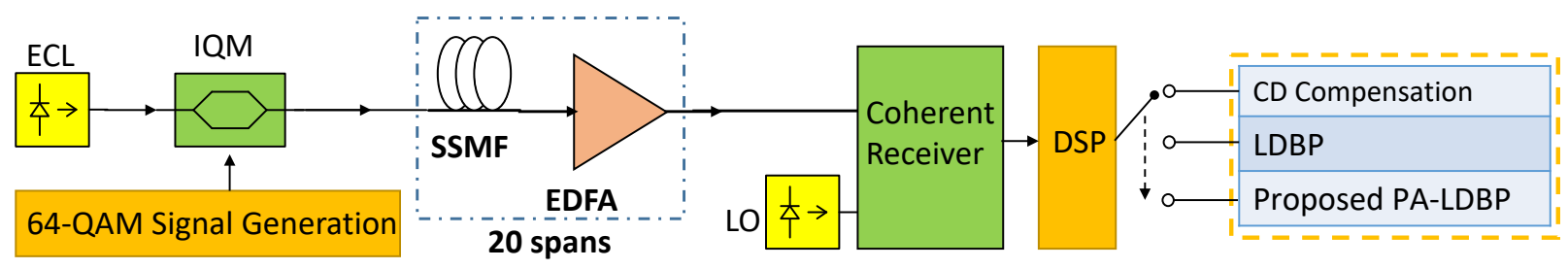

Fig. 2: Simulation system block diagram. LO: local oscillator.

LDBP as

$$
\Pi_{\mathrm{PA}-\mathrm{LDBP}}(\mathbf{x})=\sigma\left(\mathbf{C}^{(L)} \mathbf{W}^{(L)} \ldots \sigma\left(\mathbf{C}^{(1)} \mathbf{W}^{(1)} \mathbf{x}\right)\right) .
$$

The resulting structure of PA-LDBP is shown in Figure 1. The dashed-line window, given as an example, includes three neurons for nonlinearity calculation contributing to the neuron (in blue) of the next layer. Although the complexity at each step increases compared to LDBP, the introduced extra operation significantly improves the nonlinearity compensation performance, and enables a flexible structure with multi-span per step. Alternatively speaking, compared to LDBP, PA-LDBP could achieve similar performance with a smaller number of steps, $L$.

\section{NUMERICAL RESULTS AND DISCUSSIONS}

In this section, the performance of the proposed PA-LDBP is investigated through numerical simulations. The details about the simulation implementation are provided, followed by the demonstration of the simulation results.

\section{A. Simulation Setup}

1) Optical Fiber System Setup: A single carrier system, shown in Fig. 2, is employed to generate the training and testing data. A 64-quadrature amplitude modulation (QAM) signal at 32 Gbaud with a roll-off factor of 0.1 is generated to modulate an optical carrier through the in-phase quadrature modulator (IQM). The carrier is provided by an external cavity laser (ECL) with $1550.12 \mathrm{~nm}$ center wavelength. Then, the modulated signal is fed into 20 spans of standard single mode fiber (SSMF). For each span, the SSMF is $80 \mathrm{~km}$, followed by an Erbium-doped fiber amplifier (EDFA). The SSFM has an attenuation coefficient of $0.2 \mathrm{~dB} / \mathrm{km}$, a dispersion parameter of $17 \mathrm{ps} / \mathrm{nm} / \mathrm{km}$, and a nonlinear coefficient of $1.3 / \mathrm{W} / \mathrm{km}$. The EDFA has a $5 \mathrm{~dB}$ noise figure 


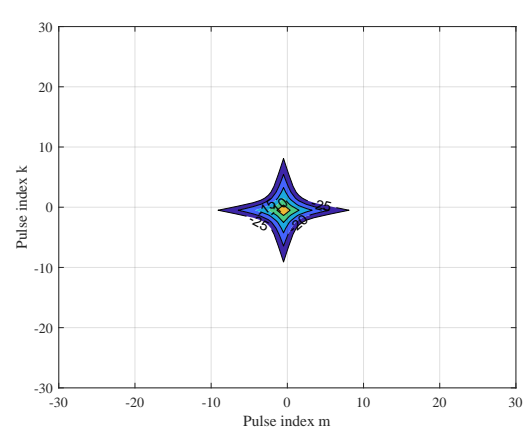

(a) $C_{m, k}$ for 1 span

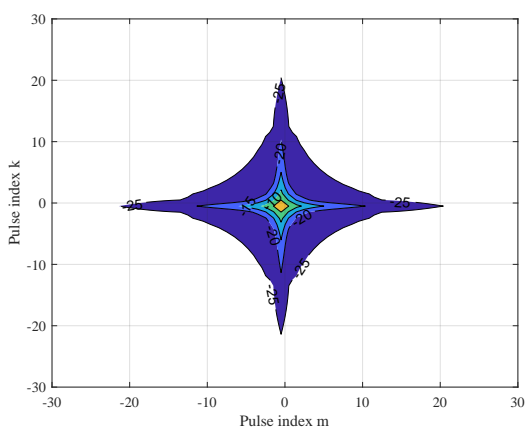

(b) $C_{m, k}$ for 2 spans

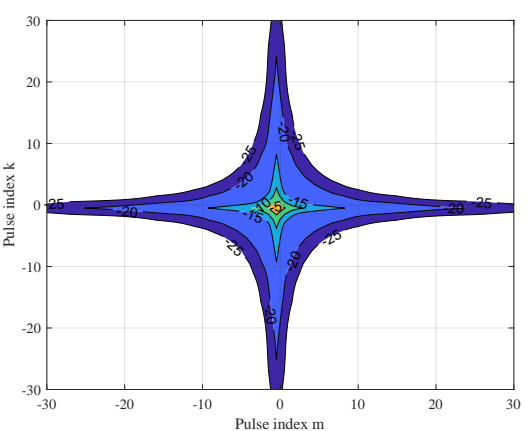

(c) $C_{m, k}$ for 4 spans

Fig. 3: perturbation coefficients contour plot at the truncation threshold of $-25 \mathrm{~dB}$ for fiber lengths of 1,2, and 4 spans, respectively.

TABLE I: FIR filter and $\mathbf{c}_{\boldsymbol{0}}$ lengths per step for PA-LDBP initialization.

\begin{tabular}{|c|c|c|}
\hline $\begin{array}{c}\text { Scenarios } \\
(\operatorname{span}(\mathrm{s}) / \mathrm{step})\end{array}$ & $\begin{array}{c}\text { FIR filter } \\
\text { lengths }\end{array}$ & $\begin{array}{c}\mathbf{c}_{\boldsymbol{0}} \\
\text { lengths }\end{array}$ \\
\hline 1 & 77 & 11 \\
\hline 2 & 149 & 25 \\
\hline 4 & 293 & 31 \\
\hline 10 & 725 & 41 \\
\hline
\end{tabular}

and $16 \mathrm{~dB}$ gain. The propagation is emulated by the SSFM with 100 steps per span. Similar to [24] and [27], carrier frequency offset and laser phase noise are not considered in the simulation. At the receiver, the processing includes static equalization and phase rotation recovery. The $Q^{2}$

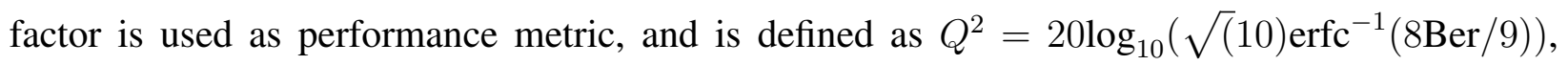
where $\operatorname{erfc}(\cdot)$ is the complementary error function and Ber is the system bit error rate.

2) Configurations with Different Number of Spans per Step: According to (9) and (11), a given pulse interacts nonlinearly with a certain number of neighbouring pulses over a distance, and this number depends on the fiber length. To investigate the flexibility of the proposed scheme, we consider four scenarios with 1, 2, 4, 10 spans per step. Accordingly, $\mathbf{h}$ and $\mathbf{c}_{\mathbf{0}}$ are initialized with fiber lengths of $80 \mathrm{~km}, 160 \mathrm{~km}, 320 \mathrm{~km}$, and $800 \mathrm{~km}$ (note that same $\mathbf{h}$ and $\mathbf{c}_{\mathbf{0}}$ are used to initialize each steps in the LDBP and PA-LDBP). For $\mathbf{h}$, the FIR filter is obtained based on the least square criterion [24], and $\mathbf{c}_{\boldsymbol{0}}$ can be computed by (9). The length of $\mathbf{c}_{\mathbf{0}}$ 
depends on the window length corresponding to a truncation threshold [16]. The truncation is to keep only the significant coefficients for complexity reduction. A truncation criterion, defined as $20 \log _{10}\left|C_{m, k} / C_{0,0}\right|=\chi$, is applied, with $\chi$ as a truncation threshold. In Fig. 3, we show the values of $\chi$ ranging from $-25 \mathrm{~dB}$ to $-5 \mathrm{~dB}$ with a step size of $5 \mathrm{~dB}$ for different spans of fiber length. Given a selected truncation threshold, the numbers of significant terms of perturbation coefficients increases with augmenting the number of spans because of the dispersive effect. In the simulation, a truncation threshold of $-20 \mathrm{~dB}$ is used, and the corresponding length of $\mathbf{c}_{\mathbf{0}}$ for the four scenarios are 11, 25, 51, and 101, respectively. This threshold value is a good compromise between performance and complexity, as the performance improvement of PA-LDBP with a smaller threshold is insignificant. Furthermore, in the cases of 4 and 10 spans per step, we found that the lengths of $\mathbf{c}_{\mathbf{0}}$ can be reduced to 31 and 41 after the training, respectively, without performance penalty. The lengths of the FIR filter and $\mathbf{c}_{0}$ to initialize PA-LDBP for the 4 scenarios are summarized in Table I.

3) Training of PA-LDBP: The PA-LDBP is implemented in TensorFlow. The input is the signal after coherent detection with 2 samples per symbol. The training data set includes 256 frames and each frame includes 2048 samples, while the testing data set includes 64 frames with the same frame length as the training data set. Therefore, the $Q^{2}$ factor is calculated from 393,216 bits. Adam is chosen as the optimizer with a learning rate of 0.001 , and the batch size is 32 .

The loss function is the mean-squared error defined as $\mathcal{L}(\mathbf{s}, \hat{\mathbf{s}})=\sum_{n=1}^{N}\left|s_{n}-\hat{s}_{n}\right|^{2} / N$, where $\hat{s}_{n}$ is the $n$th symbol after downsampling and phase de-rotation. Furthermore, the effective SNR, calculated by $10 \log _{10}\left(\mathcal{L}(\mathbf{s}, \hat{\mathbf{s}})^{-1}\right)$, is employed as an alternative performance metric to better demonstrate the DNN's convergence performance in the next section.

\section{B. Performance of the PA-LDBP}

First, we present the $Q^{2}$ factor performance of the proposed PA-LDBP and compare it to that of LDBP, as shown in Fig. 4. The performance gains of PA-LDBP over linear compensation are approximately $3.5 \mathrm{~dB}, 1.8 \mathrm{~dB}, 1.4 \mathrm{~dB}$, and $0.6 \mathrm{~dB}$, when the numbers of spans per step are 1, 2, 4, 10, respectively. Furthermore, PA-LDBP outperforms LDBP by $0.9 \mathrm{~dB}, 1.1 \mathrm{~dB}, 0.9 \mathrm{~dB}$, and 


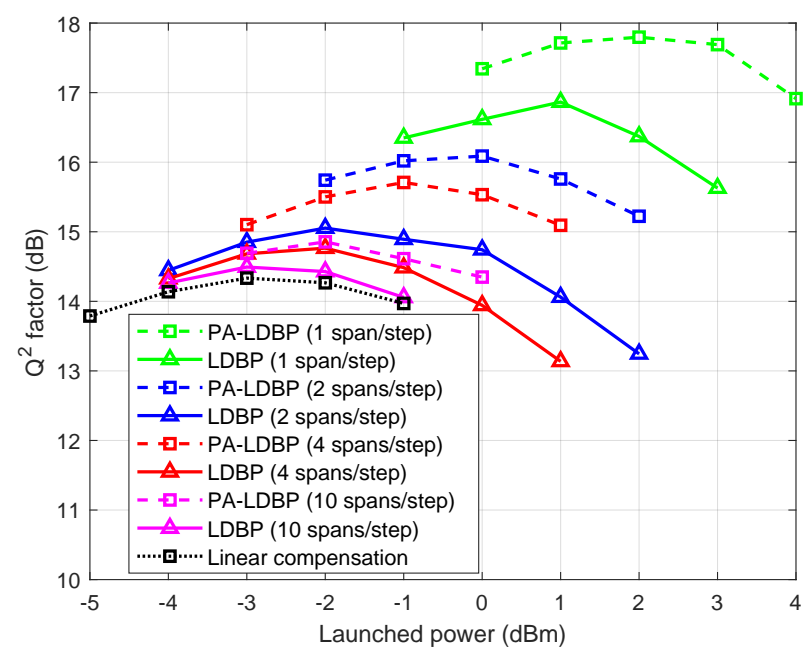

Fig. 4: Performance comparisons between LDBP and PA-LDBP.

$0.5 \mathrm{~dB}$ for the same number of spans per step, respectively. These performance improvements are expected, given the fact that PA-LDBP compensates for IXPM along with SPM at each nonlinear step. Performance gains diminish when the number of spans per step increases for both PA-LDBP and LDBP, as a large number of spans per step results in a numerical approximation error in calculating the nonlinear distortion field. However, it is noticed that PA-LDBP with 10 spans per step achieves a similar gain when compared to LDBP with 2 spans per step. The overall complexity with 10 spans per step would be much lower than the case with 2 spans per step. Details are shown in the complexity section.

\section{Initialization for $P A-L D B P$}

The initialization is critical to DNN's performance, as an inappropriate initialization could lead to slow learning or divergence. The parameter initialization for the linear steps is similar to that of the LDBP, which has been discussed in [24]. Therefore, we focus on the discussion of the initialization for the nonlinear steps. In particular, for the four scenarios, the initial FIR filter length and number of perturbation coefficients are listed in Table I. Similar to what has been observed in [24] for the linear steps, it turns out that the initialization of nonlinear step in PA-LDBP plays an important role on the convergence of the DNN. Two initialization scenarios 


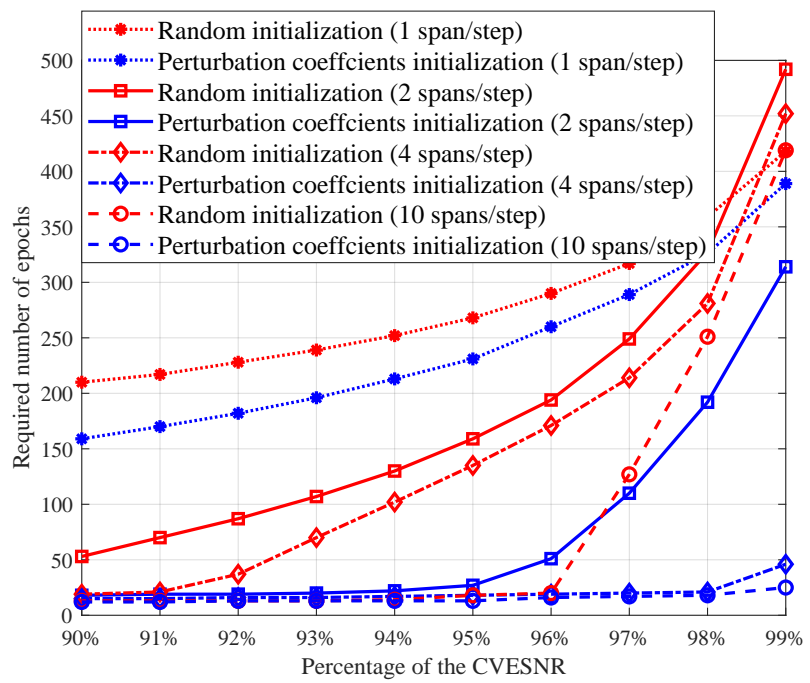

Fig. 5: Number of epochs required to achieve a certain level of the CVESNR with different initializations for PA-LDBP.

are considered: the initialization with the perturbation coefficients calculated by (9) and random initialization. For the latter case, the real and imaginary parts of all filter taps are drawn from a Gaussian distribution with zero mean and unit variance. The results are shown in Fig. 5, where the number of epochs to achieve $90 \%$ to $99 \%$ of the converged value of the effective SNR (CVESNR) is illustrated to indicate the quality and speed of convergence. Note that when the random initialization is used, 20 realizations are performed to obtain the average. As can be seen,

the initialization with the perturbation coefficients leads to a fast and good convergence of the DNN, especially when the number of spans per step is greater than one. This could be an advantage for an elastic optical network where training is required more frequently to cope with the adaptive transmission.

\section{COMPlEXITy ANALYSIS AND REDUCTION}

Complexity is crucial when designing a digital NLC technique. The complexity metric adopted here is the number of real-valued multiplications, as they typically consume significantly more 
computation resources than other operations. In this section, the complexity of PA-LDBP is investigated first, followed by two proposed methods to reduce the complexity. Then, the performance versus complexity of PA-LDBP and LDBP is provided.

\section{A. The Complexity of $P A-L D B P$}

Firstly, the complexity of LDBP is analysed as a benchmark. At each linear step, by considering that the FIR filter is symmetric, the number of real-valued multiplications per sample is 4 . $\operatorname{ceil}\left(N_{\mathrm{CD}} / 2\right)$, where $N_{\mathrm{CD}}$ is the FIR filter length and ceil $(\cdot)$ is the ceiling function. At each nonlinear step, each sample is squared ( 2 real-valued multiplications), then multiplied by $\gamma$ ( 1 real-valued multiplication), followed by a phase rotation (4 real-valued multiplications). The exponential function is implemented by a look-up table. The complexity analysis for PA-LDBP is done in a similar way, and the major difference lies in the nonlinear steps. On top of the LDBP complexity in each nonlinear step, extra $4 \cdot \operatorname{ceil}\left(N_{\mathrm{PB}} / 2\right)$ real-valued multiplications per sample are required, where $N_{\mathrm{PB}}$ is the number of perturbation coefficients at each step.

\section{B. Complexity Reduction}

We propose two methods to reduce the overall complexity of PA-LDBP, as presented below.

1) Perturbation Coefficient Pruning: Network pruning is important for memory size and complexity reduction, and aims to remove redundant weights or neurons that do not contribute significantly to the accuracy of the networks output. In [24], the CD FIR filter length can be decreased significantly through progressive pruning, which contributes a substantial complexity reduction with negligible performance penalty. More specifically, the lengths of the FIR filters after pruning are $37,51,95,251$ per step for the 4 scenarios, respectively. Compared to the values in Table I, the FIR filter lengths are pruned more than half. This enhancement is attributed to the efforts of joint filter design by the DNN: all the steps are incorporated into a multi-objective optimization problem instead of the standard least-square formulation on one step. The pruning 


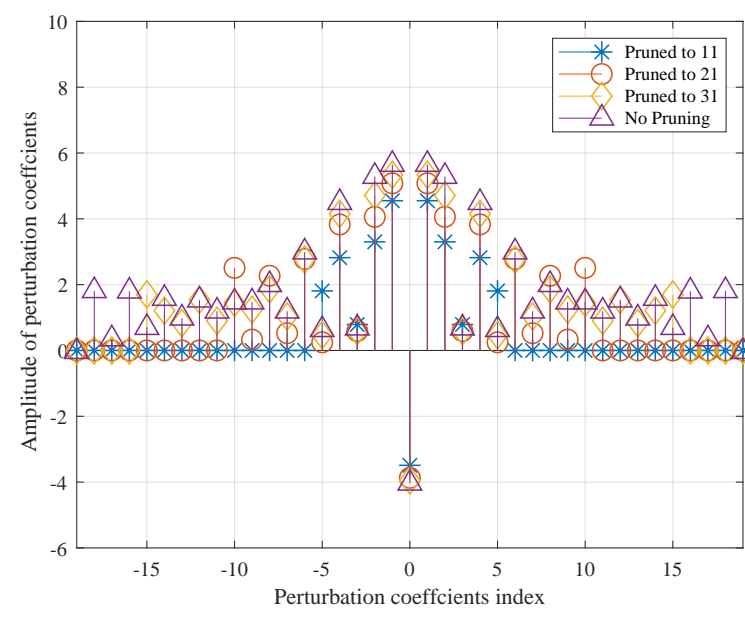

(a) $C_{m, k}$ at Step 1

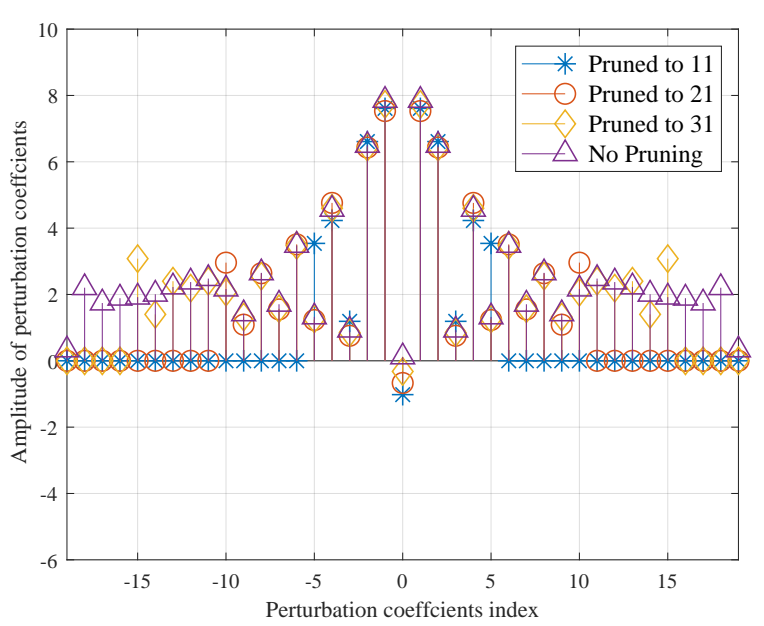

(b) $C_{m, k}$ at Step 2

Fig. 6: Prior- and post-pruning perturbation coefficients at different steps, for 10 spans per step.

TABLE II: The performance with perturbation coefficients pruning (10 spans per step at launch power $-2 \mathrm{dBm})$.

\begin{tabular}{|l|c|c|c|}
\hline $\begin{array}{l}\text { Number of perturba- } \\
\text { tion coefficient after } \\
\text { pruning }\end{array}$ & 31 & 21 & 11 \\
\hline$Q^{2}$-factor (dB) & 14.99 & 14.92 & 14.82 \\
\hline
\end{tabular}

for linear steps from LDBP can be inherited by PA-LDBP. Furthermore, the trainable parameters in the nonlinear steps, i.e., the perturbation coefficients in $\mathbf{c}_{0}$, can be pruned as well.

Illustrative examples are provided in Table II, where the performance for 10 spans per step is shown with 31,21 , and 11 perturbation coefficients, respectively. Out of these cases, no performance penalty is observed when reducing the number of perturbation coefficients from 41 to 31 . The amplitude of perturbation coefficients after pruning at each step is shown for steps 1 and 2 in Fig. 6. As can be seen, the coefficients at the two steps are not identical after training, while the symmetry of perturbation coefficients is preserved after pruning.

Pruning the perturbation coefficients is beneficial for complexity reduction with a negligible performance penalty. The reason is similar to what has been stated for the pruning strategy for the FIR filter in LDBP, i.e., the efforts of joint filter design by the DNN. 


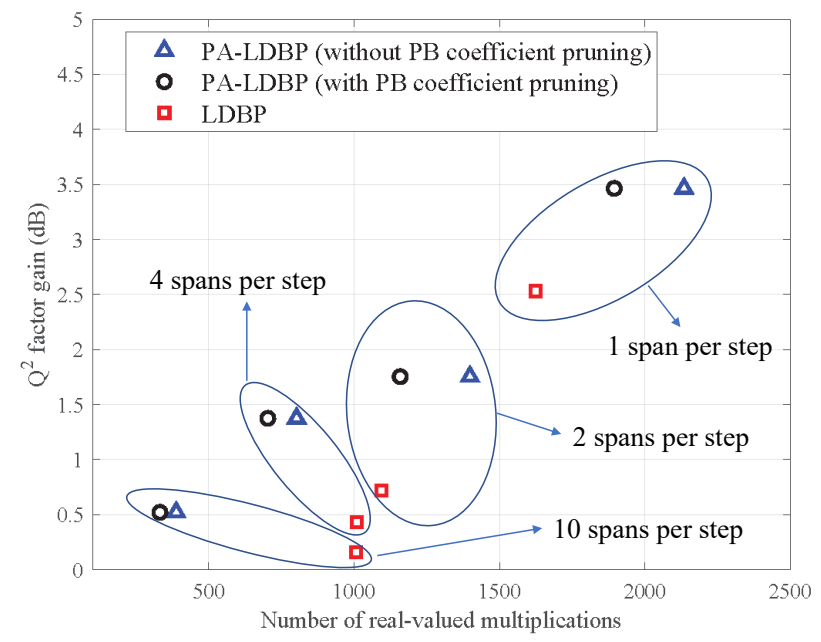

Fig. 7: Performance gain versus the number of real-valued multiplications for PA-LDBP and LDBP.

2) CD Compensation in Frequency Domain: In [24], [25], convolution operations are embedded in the matrix operations in the DNN for CD compensation, and in [27], a CNN is employed instead. These TDEs that compensate for the CD at each linear step might be efficient when the FIR filter length is relatively short. More importantly, the filter coefficients pruning is straightforward to be implemented in time domain. However, for multiple spans per step, frequency domain equalization (FDE) can be more computationally-efficient because a longer filter is required.

At each linear step, one pair of fast Fourier transformation (FFT) and inverse FFT, and multiplications with the FDE filter coefficients at the corresponding discrete frequencies are required. For processing long sequences, the overlap-and-add (OLA) method is used to segment the sequence into shorter-length blocks. Using FDE, the number of real-valued multiplications per sample is $4 \cdot\left[2 \cdot N_{\mathrm{FFT}} \log _{2}\left(N_{\mathrm{FFT}}\right)+N_{\mathrm{FFT}}\right] /\left(N_{\mathrm{FFT}}-N_{\mathrm{CD}}\right)$, where $N_{\mathrm{FFT}}$ is the FFT size in the OLA method. The complexity-optimal FFT sizes corresponding to the four investigated scenarios (from 1 span per step to 10 spans per step) are 256, 512, 1024, and 2048, respectively. In practice, we recommend to obtain the pruned TDE filter weights through training and perform FDE at the inference stage, as complexity at the inference stage is of more practical interest. 


\section{Performance versus Complexity}

Figure 7 shows the $\mathrm{Q}^{2}$ factor gain over linear compensation as a function of the number of real-valued multiplications per sample for LDBP and FDE-based PA-LDBP. Pruning of the CD filter coefficients is applied in all cases. As can be seen, for 1 and 2 spans per step, PA-LDBP without perturbation coefficients pruning obtains improved performance at the price of higher complexity. However, when the number of spans per step increases, in the cases of 4 and 10 spans per step, PA-LDBP attains an enhanced performance with a reduced complexity. Pruning for PA-LDBP reduces the number of perturbation coefficients to 5, 13, 21, and 27 per step for the 4 scenarios, respectively. We observe that this leads to a considerable reduction of complexity for 1 and 2 spans per step. With the perturbation coefficients pruning, PA-LDBP bears a similar complexity as LDBP for 2 spans per step, while attaining an enhanced performance.

\section{CONCLUSION}

A perturbation-aided machine learning scheme has been proposed for intra-channel nonlinearity compensation in coherent optical fiber communications systems. The proposed scheme improves the nonlinearity compensation at each nonlinear step by incorporating SPM and IXPM terms based on the first-order perturbation theory. This refinement has enabled a flexible restructuring of the existing LDBP technique. Furthermore, the CD compensation for multi-span per step can be performed in frequency domain, and this achieves a complexity reduction compared to the TDE-based LDBP. In addition, pruning of the perturbation coefficients has been investigated, and it has been showed that it successfully reduces the complexity. Overall, the proposed scheme attains an enhanced performance when compared with the LDBP scheme, with possibility of reduced complexity. Additionally, it provides practitioners with a flexible way to configure the compensation scheme with different numbers of spans per step.

\section{REFERENCES}

[1] S. J. Savory, "Digital filters for coherent optical receivers," Opt. Express, vol. 16, no. 2, pp. 804-817, Jan. 2008. 
[2] D. Chang et al., "Robust faster-than-Nyquist PDM-mQAM systems with Tomlinson-Harashima precoding," IEEE Photon. Technol. Lett., vol. 28, no. 19, pp. 2106-2109, Oct. 2016.

[3] S. Zhang et al., "Training symbol-based equalization for quadrature duobinary PDM-FTN systems," IEEE Photon. Technol. Lett., vol. 29, no. 5, pp. 454-457, Mar. 2017.

[4] X. Lin et al., "Joint modulation classification and OSNR estimation enabled by support vector machine," IEEE Photon. Technol. Lett., vol. 30, no. 24, pp. 2127-2130, Oct. 2018.

[5] P. J. Winzer et al., "Fiber-optic transmission and networking: the previous 20 and the next 20 years," Opt. Express, vol. 26, no. 18 , pp. 24 190-24239, Aug. 2018.

[6] X. Lin et al., "A non-data-aided OSNR estimation algorithm for coherent optical fiber communication systems employing multilevel constellations," J. Lightw. Technol., vol. 37, no. 15, pp. 3815-3825, Jun. 2019.

[7] M. Kuschnerov et al., "DSP for coherent single-carrier receivers," J. Lightw. Technol., vol. 27, no. 16, pp. 3614-3622, Jun. 2009.

[8] K. Kikuchi, "Fundamentals of coherent optical fiber communications," J. Lightw. Technol., vol. 34, no. 1, pp. 157-179, Aug. 2015.

[9] E. Ip et al., "Coherent detection in optical fiber systems," Opt. Express, vol. 16, no. 2, pp. 753-791, Jan. 2008.

[10] X. Liang and S. Kumar, "Multi-stage perturbation theory for compensating intra-channel nonlinear impairments in fiberoptic links,” Opt. Express, vol. 22, no. 24, pp. 29733-29745, Nov. 2014.

[11] A. Amari et al., "A survey on fiber nonlinearity compensation for $400 \mathrm{~Gb} / \mathrm{s}$ and beyond optical communication systems," IEEE Commun. Surveys Tuts., vol. 19, no. 4, pp. 3097-3113, Jun. 2017.

[12] X. Liang and S. Kumar, "Correlated digital back propagation based on perturbation theory," Opt. Express, vol. 23, no. 11, pp. $14655-14665$, May 2015.

[13] X. Liang et al., "Perturbation-assisted DBP for nonlinear compensation in polarization multiplexed systems," IEEE Photon. Technol. Lett., vol. 29, no. 21, pp. 1812-1815, Nov. 2017.

[14] M. Secondini et al., "Single-step digital backpropagation for nonlinearity mitigation," Photon. Netw. Commun., vol. 31, no. 3, pp. 493-502, Jun. 2016.

[15] O. S. Kumar et al., "Enhanced regular perturbation-based nonlinearity compensation technique for optical transmission systems," IEEE Photon. J., vol. 11, no. 4, pp. 1-12, Aug. 2019.

[16] S. K. Orappanparasoman et al., "Second-order perturbation theory-based digital predistortion for fiber nonlinearity compensation," J. Lightw. Technol., Jun. 2021.

[17] J. Zhang et al., "Non-data-aided k-nearest neighbors technique for optical fiber nonlinearity mitigation," J. Light. Technol., vol. 36, no. 17, pp. 3564-3572, Sep. 2018.

[18] A. Amari et al., "Fiber nonlinearity mitigation via the parzen window classifier for dispersion managed and unmanaged links," in Proc. IEEE Int. C. Trans. Opt. Netw., Jul. 2019, pp. 1-4.

[19] —_ "A machine learning-based detection technique for optical fiber nonlinearity mitigation," IEEE Photon. Technol. Lett., vol. 31, no. 8, pp. 627-630, Mar. 2019.

[20] O. Sidelnikov et al., "Equalization performance and complexity analysis of dynamic deep neural networks in long haul transmission systems," Opt. Express, vol. 26, no. 25, pp. 32 765-32 776, Dec. 2018. 
[21] S. Zhang et al., "Field and lab experimental demonstration of nonlinear impairment compensation using neural networks," Nat. Commun., vol. 10, no. 1, pp. 1-8, Jul. 2019.

[22] I. Goodfellow et al., Deep Learning. MIT Press Cambridge, 2016.

[23] C. Häger and H. D. Pfister, "Nonlinear interference mitigation via deep neural networks," in Proc. Opt. Fiber Commun. Conf., Mar. 2018, paper W3A.4.

[24] — - "Physics-based deep learning for fiber-optic communication systems," IEEE J. Sel. Areas Commun., vol. 39, no. 1, pp. 280-294, Jan. 2021.

[25] Q. Fan et al., "Advancing theoretical understanding and practical performance of signal processing for nonlinear optical communications through machine learning," Nat. Commun., vol. 11, no. 1, pp. 1-11, 2020.

[26] V. Oliari et al., "Revisiting efficient multi-step nonlinearity compensation with machine learning: An experimental demonstration," J. Lightw. Technol., vol. 38, no. 12, pp. 3114-3124, Jun. 2020.

[27] O. Sidelnikov et al., "Advanced convolutional neural networks for nonlinearity mitigation in long-haul WDM transmission systems," J. Lightw. Technol., vol. 39, no. 8, pp. 2397-2406, Apr. 2021.

[28] G. P. Agrawal, "Nonlinear fiber optics: its history and recent progress," J. Opt. Soc. Amer. B, vol. 28, no. 12, pp. A1-A10, Dec. 2011

[29] E. Ip and J. M. Kahn, "Compensation of dispersion and nonlinear impairments using digital backpropagation,” J. Lightw. Technol., vol. 26, no. 20, pp. 3416-3425, Oct. 2008.

[30] C. Häger and H. D. Pfister, "Deep learning of the nonlinear Schrödinger equation in fiber-optic communications," in Proc. IEEE Int. Symp. on Information Theory (ISIT), 2018, pp. 1590-1594.

[31] Z. Tao et al., "Analytical intrachannel nonlinear models to predict the nonlinear noise waveform," J. Lightw. Technol., vol. 33, no. 10, pp. 2111-2119, May 2014. 